\title{
A Case of Intrahepatic Gallstone Formation around Nylon Suture for Hepatectomy
}

\author{
HISAFUMI KINOSHITA, SHUICHI SAJIMA, KOTARO HASHINO, MITSUO HASHIMOTO, \\ SHINJI SATO, MASAHIRO KAWABATA, TSUYOSHI TAMAE, MASAO HARA, \\ HIROYASU IMAYAMA AND SHIGEAKI AOYAGI
}

Department of Surgery, Kurume University School of Medicine, Kurume 830-0011, Japan

\begin{abstract}
Summary: A 69-year-old female underwent left lobectomy for hepatolithiasis in February 1994. She was admitted to the Kurume University Hospital in December 1997 because computed tomography (CT) showed calcification in the porta hepatis. Ultrasonography (US) revealed a hyperechoic area with an acoustic shadow in the right hepatic duct. Dilated intrahepatic bile ducts and a mural lucent area in the right hepatic duct were noted on endoscopic retrograde cholangiography (ERC). Although the above findings suggested a diagnosis of recurrent hepatolithiasis, percutaneous transhepatic biliary drainage (PTBD) for biopsy was performed in order to rule out cancer. Biopsy showed no evidence of malignancy. Under a cholangioscope, a tip of a nylon suture was found to be protruding into the bile duct. Although a gallstone had already slipped off, the surface of the nylon suture was covered with biliary sludge. The protruding tip of the nylon suture was considered to be the nucleus of the stone. The tip was removed under cholangioscopy. Postoperative CT confirmed the absence of calcification in the porta hepatis. There has been no recurrence of hepatolithiasis after surgery. Although the formation of gallstones around the core of nylon sutures is very rare, absorbable sutures should be used during surgery of the bile duct because nonabsorbable sutures can become the nucleus of gallstones.
\end{abstract}

Key words intrahepatic gallstone, nylon suture

\section{INTRODUCTION}

The formation of gallstones around the nucleus of silk sutures used during biliary surgery has rarely been reported. Recently, a number of cases have been reported where gallstones were formed around a migrated clip used during laparoscopic cholecystectomy (LC). We report a case of gallstone formation around a protruding tip of a nonabsorbable suture (nylon thread) in the bile duct used during left lobectomy.

\section{CASE REPORT}

A 69-year-old female underwent left lobectomy for hepatolithiasis in February 1994. The postop- erative course was uneventful and she was followed up at a nearby clinic. However, she was admitted to the Kurume University Hospital in December 1997 because CT showed calcification in the porta hepatis. On admission, she was $159 \mathrm{~cm}$ in height and $54 \mathrm{~kg}$ in weight. The palpebral and bulbar conjunctivae were neither anemic nor icteric. The abdomen was flat without palpable tumors. Blood chemistry including tumor markers showed no abnormalities. US revealed a hyperechoic area with an acoustic shadow in the right hepatic duct. CT demonstrated a high attenuation nodule in the right hepatic duct (Fig. 1). Dilated intrahepatic bile ducts and a mural lucent area in the right hepatic duct were noted on ERC (Fig. 2).

Although the above findings suggested a diag- 


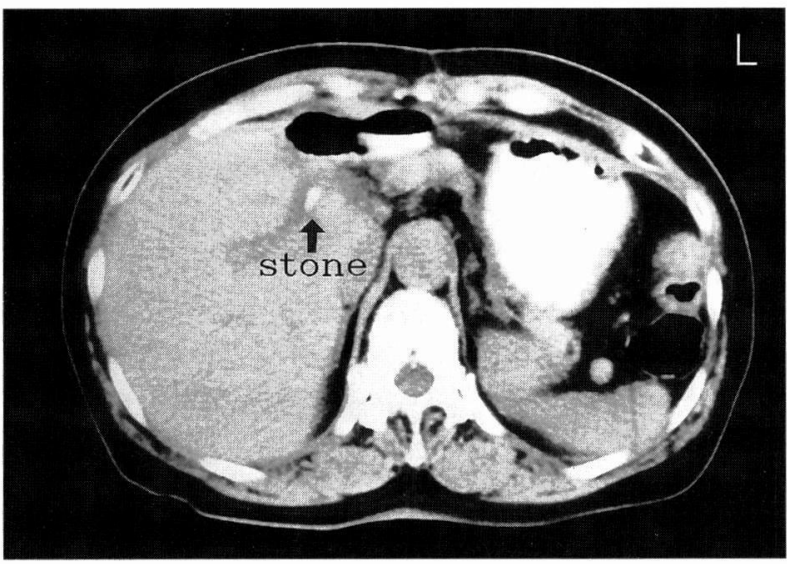

Fig. 1. CT demonstrated a high attenuation nodule in the right hepatic duct.

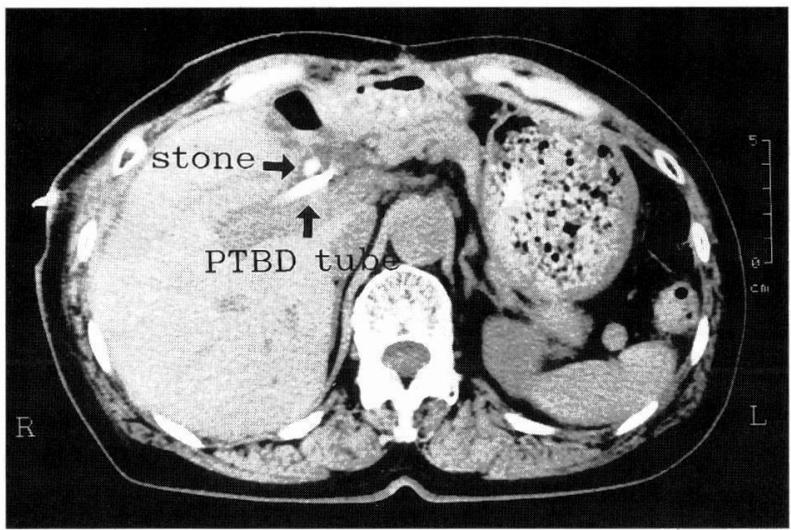

Fig. 3. CT after PTBD: A high attenuation nodule is seen just above the PTBD tube.

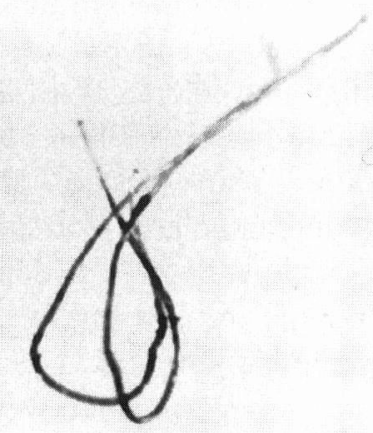

Fig. 5. The tip of the nylon suture was removed under cholangioscopy.

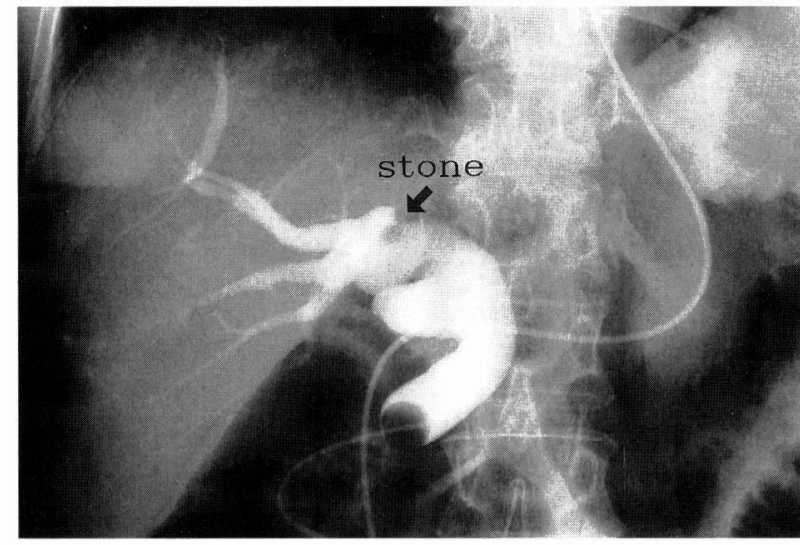

Fig. 2. Dilated intrahepatic bile ducts and a mural lucent area in the right hepatic duct were noted on ERC.

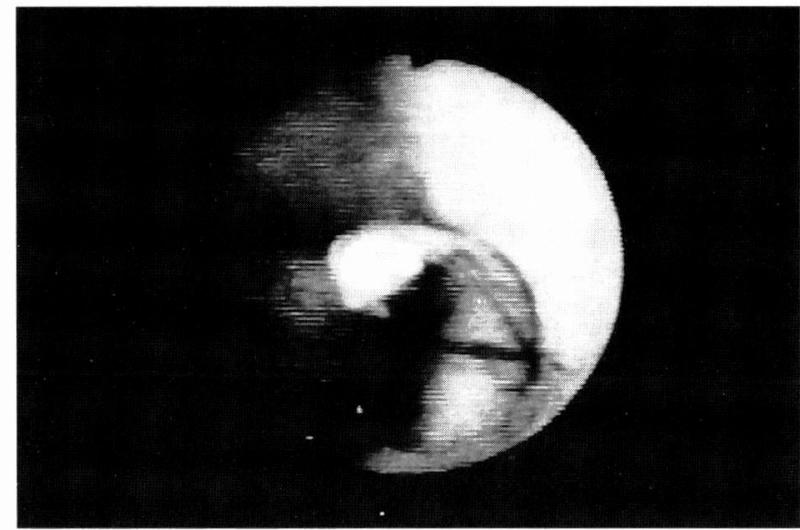

Fig. 4. Cholangioscopy : A tip of the nylon suture is seen protruding into the bile duct. Although a gallstone has already slipped off, the surface of the nylon suture is covered with biliary sludge.

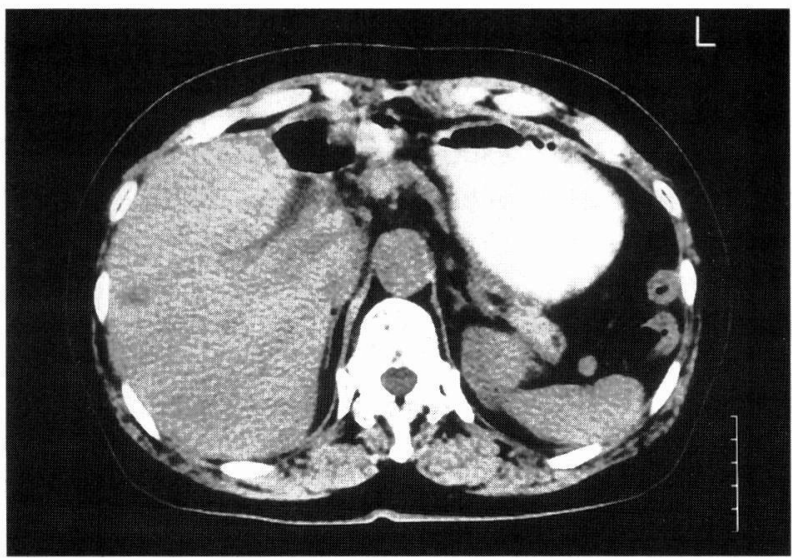

Fig. 6. Postoperative CT confirmed the absence of calcification in the porta hepatis 
nosis of recurrent hepatolithiasis, PTBD for biopsy was performed in order to rule out cancer (Fig. 3). Biopsy via the PTBD route showed no evidence of malignancy. The lumen of the biliary duct was examined using a cholangioscope inserted through the expanded PTBD route. A tip of a nylon suture protruding into the bile duct was found at the site corresponding to the lucent area on ERC. Although a gallstone had already slipped off, the surface of the nylon suture was covered with biliary sludge (Fig. 4). The protruding tip of the nylon suture was considered to be the nucleus of the stone. The tip was removed under cholangioscopy (Fig. 5). The postoperative course was uneventful. Postoperative CT confirmed the absence of calcification in the porta hepatis. There has been no recurrence of hepatolithiasis after surgery (Fig. 6).

\section{DISCUSSION}

There have been a few reports on gallstone formation around the core of a nonabsorbable silk suture used for the ligation of the cystic duct during cholecystectomy [1-3]. In recent years, laparoscopic cholecystectomy (LC) is frequently indicated for cholecystolithiasis. During LC, clips are used for ligation of the cystic duct and artery. A number of case reports have been presented on the formation of gallstones around the nucleus of those clips [4-6]. As for the mechanism of gallstone formation around foreign bodies, it is considered that foreign bodies such as nonabsorbable sutures induce inflammation around the bile duct, migrate and settle in the lumen for a long period of time. Bile stasis occurs, resulting in gallstone formation due to the deposition of calcium bilirubinate [7]. Okuyama et al. [7] reviewed 46 cases of foreign-body-induced gallstones reported in the Japanese and English literature. Gallstones were formed around the core of silk sutures in as many as 40 cases, and around other nonabsorbable sutures (perlon, cotton, thread of swab, nylon and dacron) in a few cases. In the present case, a gallstone was formed around the core of a nylon suture used for hemostasis. It is unknown whether the nylon thread migrated into the bile duct due to inflammation or if it was exposed to the lumen at the time of ligation. Nylon sutures have strong tensile strength, good extensibility and negligible tissue irritation in the body [8]. Because of the monofilament structure, nylon sutures have a smooth surface with good tissue penetration. Nylon sutures are frequently used for hemostasis because they produce no capillary phenomenon, cause negligible blood adhesion and rarely induce hemorrhage and hematomas. Although the formation of gallstones around the core of nylon sutures is very rare, absorbable sutures should be used during surgery of the bile duct because nonabsorbable sutures can become the nucleus of gallstones.

\section{REFERENCES}

1. Katsuki T, Tanikawa H, Sato S, and Nakagawa N Chlesterol gallstones with silk suture in its center in bile ducts.-Report of the two cases-. Jpn J Gastroenterology 1986; 3:2626-2629. (in Japanese)

2. Suzuki N, Takahashi W, and Sato T. Residual stones and recurrent stones. The biliary Tract and Pancreas 1983; 4:177-183. (in Japanese)

3. Koga A, Tabata M, Hisadome T, and Nakayama F Reoperations for the symptoms following biliary tract surgery : It's pathological conditions and the treatments. Jpn J Gastroenterol Surg 1983; 16:758-761. (in Japanese)

4. Asano H, Kano N, Ito $\mathrm{Y}$, Ise $\mathrm{H}$, Suzuki $\mathrm{N}$ et al. Common bile duct stone formed around vessel clip after laparoscopic cholecystectomy. The Biliary Tract and Pancreas 1993; 14:587-591. (in Japanese)

5. Takahashi H, Yokoi K, Wada M, Yamato T, Miyamoto $\mathrm{N}$ et al. A case of postoperative bile duct stone by aberrant surgical clip after laparoscopic cholecystectomy. Jpn J Gastroenterol Surg 1996; 29:85-88. (in Japanese)

6. Onghena T, Vereecken L, Dwey KV, and Loon CV. Common bile duct foreign body : An unusual case. Surg Laparosc Endosc 1992; 2:8-10.

7. Okuyama K, Takahashi T, Nagata M, Sato H, and Den N. A study of choledochal concrements formed around silk suture after cholecystectomy. The Biliary Tract and Pancreas 1981; 2:569-575. (in Japanese)

8. Postlethwait RW. Long term comparative study of nonabsorbable sutures. Ann Surg 1970; 171:892-898. 